easy as they are simulated by blobs of fat, minute lymph glands, and little, almost separate, nodules of thyroid tissue projecting from the goitre.

It is not unusual for the pathologist to find one or more parathyroids in the removed gland, and for there to be subsequent manifestations of insufficiency. Occasionally a patient will complain of "pins and needles" feelings, and show circumoral pallor, both features clearing quickly as if a remaining parathyroid or part thereof had taken a little time to compensate for those lost. Only very rarely does this go on to tetanic spasms of the hands and feet. The slight cases, the average ones, get better after taking a few glasses of milk. In more severe instances large doses of calcium lactate, up to 15 grammes a day, are necessary, and rarely one has to give Io c.c. of a 20 per cent solution of calcium chloride intravenously. In exceedingly rare instances the administration of parathormone is indicated.

\section{Recurrent Laryngeal Nerve Injuries.}

These are most commonly due to forcible stretching of the nerves and their occurrence reinforces the plea for gentle handling. But a ligature tied around an inferior artery too near to the gland may include the nerve, or a clamp may inadvertently be put on it, or, occasionally, it may be torn in displacing a posterior nodule. It is suggested by some that the nerve should always be identified and kept clear of; others, and they are more numerous, prefer not to look for it and think there is a risk of injury in the process of identification. The results of injury are hoarseness, due to unilateral incomplete and complete lesions, marked dyspnoea requiring tracheotomy in bilateral incomplete lesions, and loss of voice in bilateral complete lesions. In the first mentioned, the only one to be-met with if reasonable care be taken, there is usually, though not invariably, a compensation on the part of the opposite cord which allows of a great deal of recovery of normal voice.

\section{Convalescence}

The patient's pronounced well-being a fortnight after an adequate operation should not mislead either her or the surgeon as to the necessity for further convalescence. Too speedy a return to work or to the worries and cares of managing a household, especially if there are young children, is a potent cause of only partial success. Insistence, therefore, should be made upon a further spell of at least three weeks' convalescence, a period which may with advantage be increased where there has been visceral disease, where the toxicity has been pronounced, and where employment or home conditions do not permit of a gradual return to normal. Fairly frequent attendances at $a \frac{2}{2}$ follow-up department in the first year are neces- $\frac{\widehat{D}}{\mathrm{O}}$ sary. The nervous features of the disease are not $\stackrel{a}{c}$ always so completely controlled by operation as the metabolic ones, and they can be helped a great os deal in a routine check by the surgeon in which 0 increase in, or maintenance of, weight and other o general features are pointed out.

\section{THE HEART IN THYROID DYSFUNCTION}

\author{
By CORNELIO PAPP, M.D. (Milan) \\ (Clinical Assistant, National Hospital for Diseases \\ of the Heart)
}

Heart failure caused by the two extremes of $\mathrm{cr}$ thyroid dysfunction, toxic goitre and myxoedema, $ᄋ$ holds an almost unique position among chronic heart diseases insofar as it may be amenable to 7 complete and lasting cure. Cardiac signs may be $\underset{\mathbb{D}}{\stackrel{D}{C}}$ predominant in both conditions, yet digitalis is unreliable in toxic goitre and useless in myxoedema. These are the main facts which make the recognitio $\overrightarrow{0}$ of heart affections due to thyroid dysfunction $\frac{5}{8}$ है extremely important.

\section{The Heart in Toxic Goitre}

Toxic goitre affects the heart indirectly through the enhanced metabolism which causes cardiac $\frac{\mathrm{O}}{\mathrm{D}}$ fatigue, and directly by its-toxic effect on the heart muscle.

The great oxygen consumption of the tissues (50-100 per cent above normal) calls for an increased oxygen supply. The heamodynamic adjustments providing for this are an increase in the cardiac output, in the circulating blood volume, and in the velocity of circulation, while the arterio- 3 venous oxygen difference remains normal. The accumulation of metabolites is regarded as the cause of peripheral vasodilatation, which in the thyroid gland may assume the degree of arterio- $\frac{9}{5}$ venous shunt, and is thus responsible for the $\frac{}{0}$ greatly augmented venous return. The heart deals with the increased venous return by further $\tilde{N}$ raising its output (i.e. minute-volume), which in thyrotoxicosis of average severity becomes almost doubled. This is accomplished by tachycardia $\omega$ while the stroke volume remains but slightlyo affected. Tachycardia in thyrotoxicosis was firste thought to be a compensatory mechanism. But it is uneconomical from the point of view of cardiac + fatigue and unusual as cardiac adjustment, since $\frac{T}{0}$ 
in other conditions of increased venous return (i.e. arterio-venous aneurysm) the rise in minute volume is effected through increase in stroke volume. Besides, variations in tachycardia are wholly disproportionate to the momentary oxygen need of the body (Yater, I932), and tachycardia far from being beneficial may be the cause of death in thyroid crisis (Harris et al., I939), and. in thyroid fed animals (Rasmussen, I94I). It is the direct result of the toxic thyroid secretion on the myocardium as shown by experiments on thyrotoxic rabbits whose hearts continued to beat at an increased rate after complete isolation (Lewis and McEachern, I93I).

The heart muscle, besides providing for an increased circulation, is itself involved in the general metabolic changes. Its oxygen consumption is increased, the glycogen content is diminished, and there is an accumulation of lactic acid in it (Andrus, I932). Thus the greatly and continuously increased work of the heart, equivalent to that required normally under physical exercise, is accomplished under unfavourable metabolic conditions with its glycogen reserves depleted.

\section{Pathological findings.}

On post mortem examination slight to moderate cardiac hypertrophy,. mostly of both ventricles, can be found in about 50 per cent of the series (Parkinson and Cookson, I93I; Kepler and Barnes, I932). With great reduction of the body weight it may not be apparent, but even then the heartbody weight ratio is increased. Gross hypertrophy is exceptional, for peripheral vasodilatation eases the strain of the left ventricle, and tachycardia with its short diastole inhibits the filling of both ventricles. Age and duration of the disease increase the incidence of cardiac hypertrophy.

Thyrotoxicosis does not produce any specific lesions of the myocardium, and no microscopical changes have been detected which were not equally present in carefully selected controls. There was, however, a relatively higher incidence of myocardial fibrosis, endocardial sclerosis, and cellular infiltration in the thyroid series (Weller et al., I932; Rake and McEachern, I932). The incidence of failure in these was about I5 per cent; in two-thirds of these it could be related to additional cardio-vascular affections, while in one-third the only cause for it was thyrotoxicosis. Age and duration of the disease increased its incidence. Heart failure due to thyrotoxicosis alone, before the age of thirty-five, is most exceptional, and was found only once among 178 post mortem instances (Kepler and Barnes).

While the pathological findings emphasise the importance of superadded cardio-vascular disease -infection, hypertension, physiological wear and tear, and advancing age-as the cause of heart failure in thyrotoxicosis, no pathological explanation is forthcoming about the reason of failure in uncomplicated instances. This led many to believe that heart failure due exclusively to thyrotoxicosis is non-existent, and that superadded lesions play the decisive part in producing it. Admittedly failure is uncommon under forty years of age in uncomplicated cases, yet its existence cannot be denied. The fact that it can be cured by subtotal thyroidectomy obviously excludes gross anatomical changes as its cause. Cardiac fatigue, toxaemia, metabolic changes in the heart muscle bring about functional changes in it which may impair its contractility to a degree of failure. Structural changes appear only later and are increased by superadded cardiovascular disease of advancing age, to which the thyrotoxic heart seems to be particularly receptive.

\section{Clinical findings.}

These were fully described by Goodall (I920, I924); but little remains to be added to complete his observations.

A distinction is very often made between the cardiac signs occurring in Graves' Disease (i.e. primary thyrotoxicosis) and those in toxic adenoma (i.e. secondary thyrotoxicosis). From the cardiacstandpoint such a distinction is. immaterial since in both conditions the heart symptoms are the same. The cardiac condition, however, becomes a? greater diagnostic problem in secondary thyrotoxicosis, where a low degree of toxaemia exists and thyroid signs can easily be overlooked. These patients also belong to an age group where cardiovascular changes are to be expected; they are indeed predominant, and not easily "demasked" (Hay, I936) as thyrotoxic.

Among subjective complaints palpitation is the most frequent; it can be referred to both tachycardia and overaction of the heart, and it is probably the latter which is more important in its causation. Dyspnoea of effort is fairly common and is found independently of heart failure. It may be due to the need of hyperventilation to cover the increased oxygen requirement (Fishberg, I940). Angina pectoris is seldom present; when it is, it is due to complicating coronary arteriosclerosis which may be of such a slight degree that it would not produce cardiac pain in a normally acting heart.

Objective signs.-Tachycardia, though common in many other conditions, is so constant that its absence almost excludes thyrotoxicosis. The resting pulse rate is usually 90-120, though a rate up to I80 is nothing exceptional. The pulse is of a collapsing quality, similar to that in aortic 
incompetence. The rate is extremely labile and mounts quickly under excitement, infections, and exercise. In this respect it is similar to neurogenic tachycardia; the difference lies in the sleeping pulse rate which in thyrotoxicosis is an average of 20 beats higher than in neurogenic tachycardia (Boas, I932). A certain parallelism exists between basal metabolic rate and resting pulse rate, though not to such an extent that even approximate calculations are possible. Occasional irregularities are due to extra-systoles, and these with a high pulse rate are almost diagnostic; if the pulse is completely irregular auricular fibrillation is present. A slight to moderate rise in systolic blood pressure can be observed in many patients with the diastolic unmodified or raised, causing an increase in the pulse pressure. Similar changes are also frequent in neuro-circulatory asthenia. In addition, thyrotoxicosis can exaggerate an existent or latent hypertension.

\section{Cardiac signs.}

The thyrotoxic heart is tachycardic and overacting, and the cardiac signs are those to be found in other conditions causing overaction and tachycardia, i.e. neuro-circulatory asthenia. The apex beat is diffuse, the impulse is forcible, the whole chest wall may vibrate under the influence of the tumultuous heart action. Increased pulsation can be felt and seen over the carotid arteries and abdominal aorta. The first sound is often loud, short and slapping, the pulmonary second accentuated. Systolic murmurs due to cardiac overaction are frequently heard in the mitral and pulmonary areas. If the heart enlarges the apical systolic murmur may be due to a functional mitral incompetence, and differential diagnosis, on clinical signs alone, from a mitral lesion which may co-exist, may be difficult.

\section{Radiological signs.}

About half the cases show enlargement of the heart, and this in itself distinguishes it from neuro-circulatory asthenia. The degree of enlargement depends on the severity and duration of symptoms, on co-existent auricular fibrillation and heart failure, and on additional cardiovascular disease (i.e. hypertension). The left ventricle enlarges more, but some prominence of the right auricular border often exists. A distinct prominence of the pulmonary arch gives the heart a characteristic shape in about half the patients comprising those without cardiac enlargement (Fig. IA). It is a fairly early sign, and is due to a distension of the pulmonary artery-confirmed by Parkinson and Cookson on post mortem examination-probably less resistant to cope with the increased venous return. An additional radio-

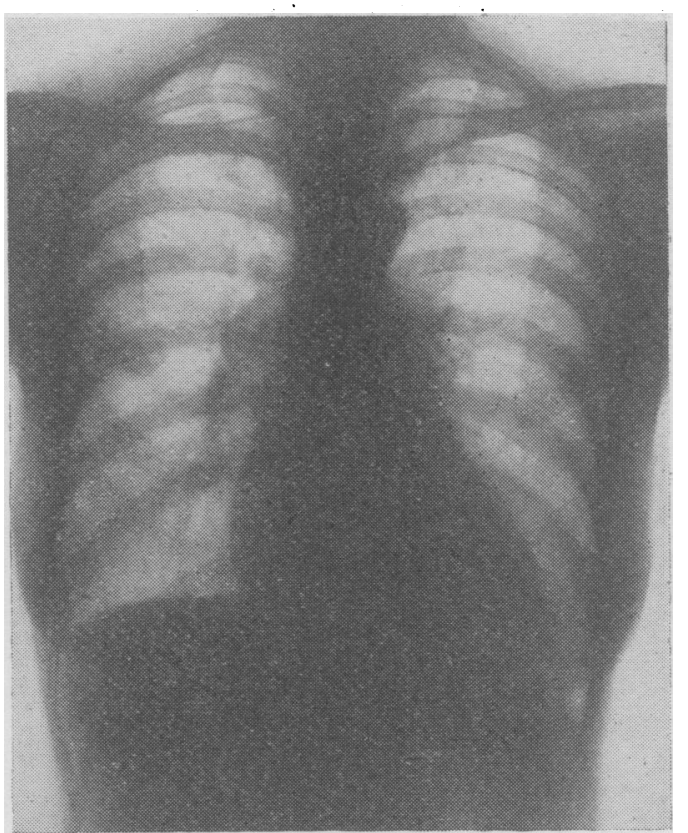

FIG. IA.-Thyrotoxicosis, normal rhythm, hypertension. Anterior view shows prominent pulmonary artery and left ventricle.

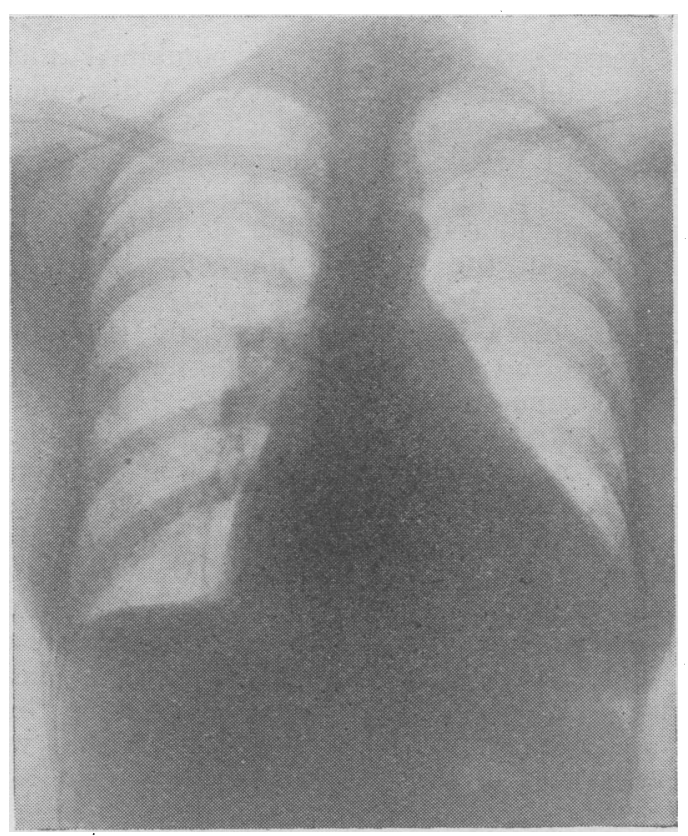

FIG. IB.-Same case four years later, auricular fibrillation and heart failure present. General enlargement, straight left border, prominent right auricle, little pulmonary congestion.

logical sign of right heart strain is the frequent prominence of the superior vena cava, to be found in some instances without failure. The shape of 


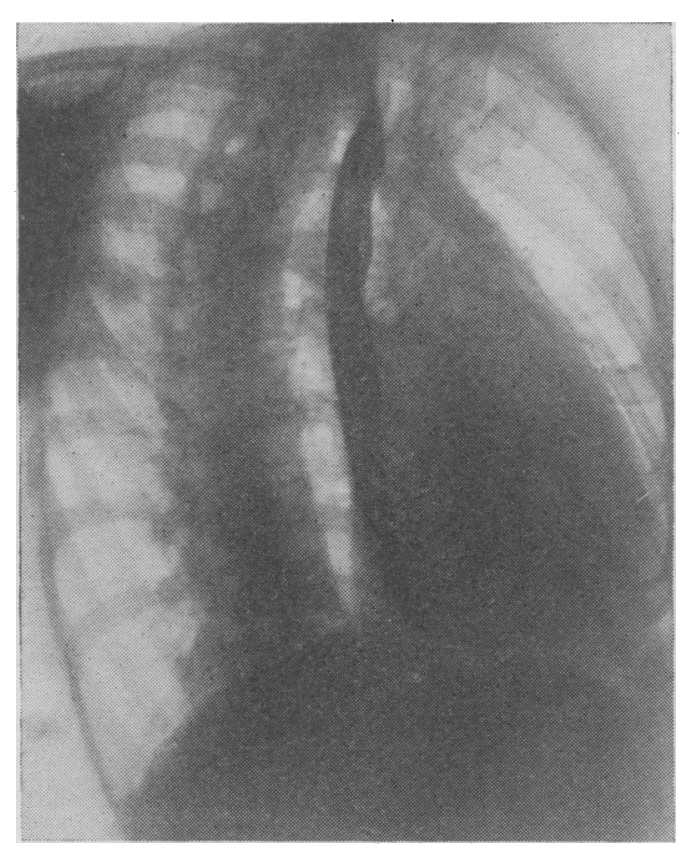

FIG. 2.-Thyrotoxicosis, auricular fibrillation present. Right oblique view, barium in the oesophagus; course straight, no left auricular enlargement. Pulmonary artery prominent.

the heart resembles that of combined mitral stenosis and aortic incompetence. A distinguishing feature, however, is the lack of left auricular enlargement in the right oblique position. Even with auricular fibrillation, when cardiac enlargement is pronounced, the left auricle takes but very little part in the general enlargement (Fig. 2). With the advent of cardiac failure the typical shape is lost; the enlargement of the conus straightens out the left heart border, the right auricle becomes greatly prominent, and the triangular shape of the heart is that found in heart failure from other causes, except from hypertension. Pulmonary congestion, however, is not such a prominent feature as it is in heart failure due to mitral stenosis or hypertension (Fig. IB).

Reduction in the size of the heart after subtotal thyroidectomy, sometimes quite pronounced, occurs in almost all patients whose congestive heart failure is relieved by it, and in some of those without heart failure, but with fibrillation, who revert to normal rhythm after the operation. In those with an enlarged heart and normal rhythm before the operation, the heart may either become smaller and the characteristic prominence of the pulmonary artery disappear, or the heart may enlarge after the operation. The latter happens in coincident heart disease, after rapid gain in weight, in post-operative myxoedema, and also for unknown reasons (Menard and Hurxthal, I932).

\section{Electrocardiographic signs.}

Tachycardia, auricular fibrillation, extrasystoles and myocardial changes are the features of the electrocardiogram in thyrotoxic heart disease. There is nothing characteristic in the electrocardiographic features singly, but the combination of some may result in records more suggestive of thyrotoxicosis than of any other heart disease. High-rate resting tachycardia, I40 or over, is rarely of neurogenic origin (Fig. 3), and if associated with

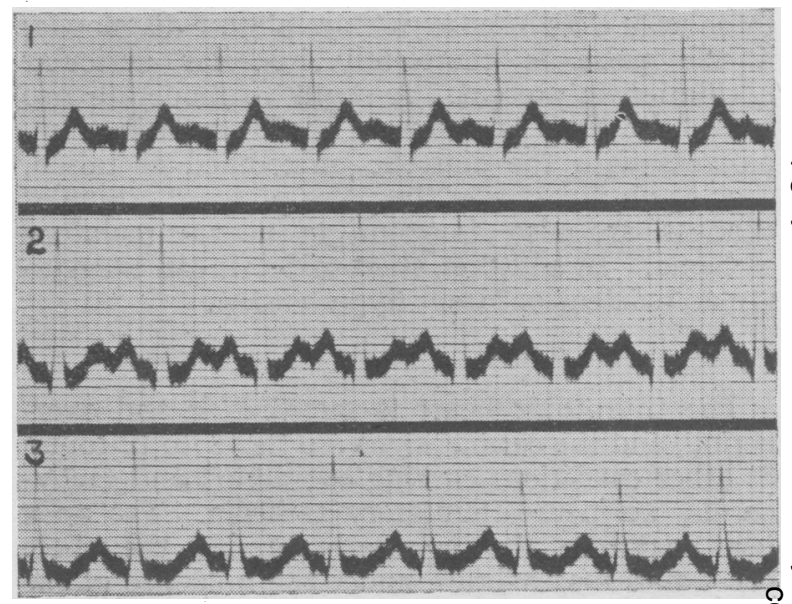

FIG. 3.-Thyrotoxicosis, sinus tachycardia, rate $I 65$.
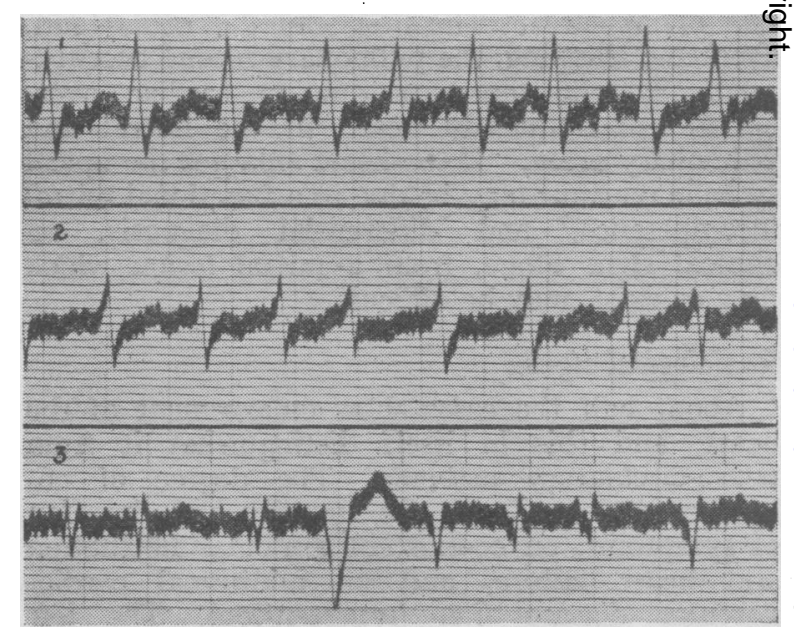

FIG. 4.-Thyrotoxicosis, auricular fibrillation, rate 220 . Ventricular extrasystole in lead 3.

extrasystoles (in 3-ro per cent of cases), becomes fairly typical for thyrotoxicosis. The same is true for high-rate auricular fibrillation with 2 extrasystoles (Fig. 4). If these extrasystoles arise after digitalis, and if other signs of digitalis action $\mathbb{D}$ can be detected on the record (i.e. depressed R-T interval), without great reduction in rate, the 


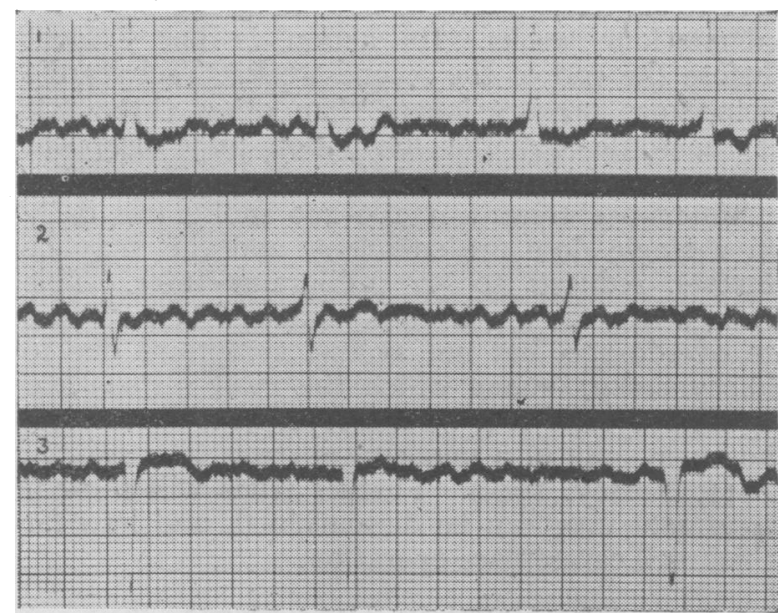

FIG. 5A-Thyrotoxicosis, hypertension, and mitral stenosis. Auricular fibrillation persisting for at least two years, controlled by digitalis at a rate of 68 .

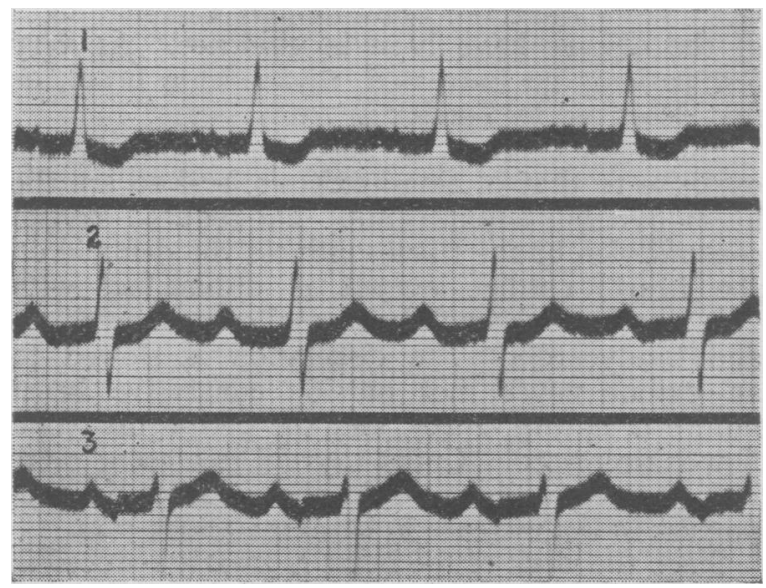

Fig. 5B. - Same case. Sinus rhythm restored after two weeks' iodine. P-R 0.30 sec., bifid Pr, broad P2, diphasic $\mathrm{P}_{3}$. Depression of R-TI due to digitalis.

electro-cardiogram becomes even more suggestive. Increase in $\mathrm{P}_{2}$ and $\mathrm{P}_{3}$ amplitude with a rate of I20 or over, may be due to superimposition of $\mathbf{P}$ and $\mathrm{U}$. But it also occurs in about one-third of all records at a slower rate and is due to right auricular strain, and so substantiates the suggestion made on radiological grounds that the right heart feels the increased cardiac fatigue more than the left. The increase in amplitude of the other waves, particularly of $T$, which was so much insisted on earlier, is most unreliable. Though its height can be increased by thyroid administration and reduced by operation, many factors are present which may reduce its voltage in thyrotoxicosis. Left axis deviation and myocardial changes are the usual ones, but apart from these, $T_{2}$ and $T_{3}$ inversion is said to be observed, as in neuro-circulatory asthenia, in young thyrotoxic patients for unknown reasons (Graybiel and White, I935). These and the other changes frequently disappear after operation.

Lesions of the conducting system are exceptional. Though the P-R interval may be prolonged in relation to the existing tachycardia, atrio-ventricular block rarely occurs, and if it does, it is more likely to be due to superadded infection or arteriosclerosis.

Auricular fibrillation is the typical arhythmia of thyrotoxicosis, and thyroid toxaemia is as much responsible for it as for tachycardia. Twenty per cent of all cases of auricular fibrillation are due to toxic goitre, and I5-22 per cent of all cases of thyrotoxicosis fibrillate. It is exceptional under the age of 30 , uncommon between $30-40$, while it becomes increasingly frequent over 40 , so much so that 80 per cent of all patients with thyrotoxicosis fibrillate when they are over 6o. Males are more prone to it than females: while the sex incidence of toxic goitre is one male to five women, the incidence of fibrillation is $\mathrm{I}: 3$. Apart from age, the duration of the disease is an important causative factor, while the intensity of toxaemia in the young, as measured by the basal metabolic rate, has but little bearing upon it. This explains its greater incidence in secondary thyrotoxicosis? Auricular fibrillation may be either transient oro permanent, and if the latter, it is sometimes? observed for the first time during the increased toxaemia present for I-2 days after the operation.

Auricular fibrillation is directly responsible for cardiac enlargement and almost all with permanent auricular fibrillation have some degree of enlargement. It is the forerunner of congestive heart failure; 90 per cent of the toxic goitre patients in heart failure are fibrillating.

Thyrotoxicosis is the only known cardiac condition in which auricular fibrillation may revert to normal rhythm by other means than quinidine or digitalis. Its direct dependence upon toxaemia is shown by the fact that relief from it, either by operation or by iodine, may reinstate sinus rhythm even when other factors responsible for auricular fibrillation, i.e. mitral stenosis, are present (Fig. 5A and B). Operation alone is responsible for the return of sinus rhythm in 50 per cent (Joll, I932); if quinidine is employed after operation the rate rises to over 80 per cent (Dunhill, I937), or even as high as 96 per cent (Anderson, I932).

\section{Treatment.}

Since the introduction of iodine as a preoperative measure, the mortality rate after subtotal thyroid- 
ectomy in hands of experienced surgeons has fallen from I5 per cent to I per cent (Joll, I932; Dunhill, I937; Linnell and Keynes, I939). No wonder that surgical treatment with its brilliant and lasting success has almost entirely replaced medical treatment.

Before the operation, patients who are in failure, or those with rapid auricular fibrillation, in addition to Lugol's Iodine ( $\mathrm{m}_{5}-\mathrm{m}_{\mathrm{o}} \mathrm{b}$ bis to t.i.d., for 2-3 weeks), should have digitalis as well. The digitalis tolerance in thyrotoxic patients is increased to more than double (Barker et al., I932), and higher dosage than usual can safely be employed. Grs. $I_{\frac{1}{2}-2}$ of the powdered leaf tablets can be given t.i.d. until mild symptoms of intoxication appear; even so in those with rapid pulse the rate seldom falls to less than 90. After the operation both digitalis, in reduced doses, and iodine should be kept up for one week. If auricular fibrillation does not disappear the fifth day after operation, quinidine should be given in divided and increasing doses, until grs. 30 are being reached the sixth day; as soon as normal rhythm reappears the drug must be stopped. If auricular fibrillation persists the same course can be repeated after one week's interval.

In cardiac patients so prepared the operative risk may be slightly higher (2-3 per cent, Slesinger, I939), than in those without cardiac complications, though in Likoff and Levine (I943) series of ninety-nine cases it was nil, while in those without heart involvement it amounted to 2.6 per cent. Recovery after successful operation is lasting, and follow-up series after many years showed no permanent cardiac damage which could have been related to thyrotoxicosis (Read, I932). In addition to the thyrotoxic heart symptoms, others due to coexistent cardio-vascular disease (angina pectoris, hypertension) may also benefit by the operation which relieves the heart from an additional strain.

In those unwilling to undergo surgical treatment, in children, or in slight cases iodine should be given a fair trial. Given in the same dose as for pre-operative treatment and during alternating fortnights, results sometimes are surprisingly good in primary as well as in secondary thyratoxicosis.

New drugs, preventing the synthesis of diiodotyrosine in the thyroid gland, and therefore highly promising in the treatment of thyrotoxic heart affections are thiourea and thiouracil. The latter has already replaced the former because of untoward side effects (halitosis, conjunctivitis, and vomiting). Patients taking iodine must be left for about a month without it, otherwise thiouracil may not be effective. The dose of thiouracil for initial treatment is roo milligrams five to six times a day, or 200 milligrams t.i.d., for about four weeks. Its action is not evident before the end of the second week. Tachycardia sometimes persists longer than other thyrotoxic signs, while auricular fibrillation may disappear with or without the help of quinidine. For maintenance treatment 100-200 milligrams daily in a single dose are sufficient, and this may be further reduced to 50 milligrams later. This dosage may be a guarantee against ill-effects due to overdosage, but not against symptoms due to idiosyncrasy, among which the most serious are agranulocytosis and thrombocytopenia, occurring, however, only in a minority of cases (Himsworth, I944; Nussey, I944). Further trials will show if treatment ever can be discontinued, and how far thiouracil can replace operation.

\section{The Heart in Myxoedema}

Myxoedema is much less of a cardiac problem than thyrotoxicosis for it is uncommon, and cardiac symptoms develop only in untreated cases years after the unmistakable external signs of the disease have been in evidence. As very few patients go untreated, myxoedema heart, as first described by 7. ondek in I9I8, is rare.

The metabolism in myxoedema is exactly the opposite to that of thyrotoxicosis, and so are the haemodynamic adjustments. The reduced oxygeno consumption of the tissues reduces the demand on the heart; cardiac output is diminished with 20-50 per cent through both smaller stroke volume and bradycardia. The circulation is slowed to an extent never met outside congestive heart failure (Fishberg, I940), and the peripheral blood flow is decreased. Under thyroid treatment there is a progressive increase in these values, in proportion to the increase in basal metabolic rate.

Since the work of the heart is greatly reduced in myxoedema, the reason for cardiac enlargement and ultimate failure has to be sought outside these metabolic conditions.

The meagre pathological evidence shows that myxoedema predisposes to arteriosclerosis, and it is believed by many that the heart changes are primarily due to it. If this were true, failure and cardiac enlargement would not be reversible as they are in myxoedema. Besides, there is evidence that deficiency in thyroid secretion reduces the functional capacity of the heart muscle, as it reduces the strength and tonus of voluntary muscle, and thus may produce dilatation of the heart. Accumulation of myxoedema fluid, causing interstitial oedema in the heart muscle as proved by post mortem findings, may play an additional part in producing heart failure.

The incidence of heart failure in myxoedema is variously estimated in different series. The two 
extremes are represented by Fahr (I925), who among I7 cases found congestive failure in I3, and Willius and Haines (I925), who among I62 cases did not find it once. The difference in the severity of hypothyroidism and in the number of previously untreated cases may account for the discrepancy. However, heart failure due to myxoedema at present must be rare, and very few instances have been reported in the last decade (Hallock, I933; Gant, 1935).

Subjective complaints.-Dyspnoea, when it precedes failure, has been related to decreased vital capacity. Anginal pain is frequently complained of, and is due to coexistent coronary arteriosclerosis, and sometimes to anaemia. Thyroid extract may aggravate the pain, lessen it or leave it unaffected. For many patients an optimum dosage can be found by which the coronary blood flow and the cardiac output increases to an extent compatible with the narrowing of the coronary arteries (Peel, I943).

\section{Clinical signs.}

Bradycardia with or without failure is almost always present. The systolic blood pressure is said to be low, the diastolic raised, and the pulse pressure small, but frequently coexisting hypertension makes it difficult to establish a rule. The heart sounds are faint and distant. Cardiac failure when present shows the usual signs; effusions in the serous cavities appear early and are due as much to myxoedema as to failure.

\section{Radiological signs.}

No heart changes can be detected in patients under thyroid treatment which could not be referred to complicating cardio-vascular disease. Untreated patients may also show a normal heart, or moderate or gross cardiac enlargement according to the severity of the disease and its duration. Cardiac enlargement is generalised and comprises all four chambers of the heart; sometimes it is exaggerated by pericardial effusion (Feasby, 1940) The heart shadow has no characteristic shape, and its pulsations are sluggish. The only characteristic of myxoedema heart is its reduction in size under thyroid treatment. Lerman (I932) observed in $\mathrm{I} 4$ of his cases a reduction in the transverse diameter of $2-7 \mathrm{~cm}$., and in 6 of $\mathrm{I}-2 \mathrm{~cm}$., and others confirmed it. The maximum reduction is reached in about two months' treatment. 'Accordion heart" (Barron, referred by Hallock) is the fitting name characterising this behaviour.
Electrocardiographic signs are early and often precede cardiac enlargement. Flat or inverted TI and T2 are the commonest findings. In many there is also low voltage in all leads; $P$ and $T$ are often inconspicuous. The P-R distance is sometimes prolonged, and QRS may be widened. Changes to normal are observed as soon as after a fortnight's treatment, but it takes about eight weeks before the electrocardiogram becomes completely normal. It is believed that these changes are due to cardiac oedema and to compression of the muscle bundles by it.

\section{Treatment.}

Digitalis is ineffective, and treatment consists in oral administration of thyroid extract. It is the rule to start with small doses to test the patient's sensibility, and gr. $\frac{1}{2}$ t.i.d. of the B.P. tablets is given for the first week, then it is increased to gr. I t.i.d. The maintenance dose has to be found out in each patient; in cases of moderate severity gr. $\frac{1}{2}$ bis to t.i.d. will be effective. Thyroid may be discontinued for a fortnight, or even for longer. In one of Fahr's (1932) patients it took eight months for signs of cardiac failure to reappear, when he was left without treatment.

\section{REFERENCES}

ANDERSON, J. P. (1932), Amer. Heart J., 8, 128.

ANDRUS, E. C. (1932), Amer. Heart $J ., 8,66$

BARKER, P. S., BOHNING, A L., and WILSON, F. N. (1932) Amer. Heart J., 8, $12 \mathrm{r}$.

BOAS, E. P. (r932), Amer. Heart $J ., 8,24$.

DUNHILL, Sir T. (1937), Trans. Med. Soc., 60, 234.

FAHR, G. (1925), J. Amer. med. Ass., 84, 345; (1932), Amer. Heart J. 8, 9 r.

FEASBY, W. R. (1940), Amer. Heart J., 19, 749.

FISHBERG, A. M. (1940), Heart failure, London.

GANT, J. C. (1935), New Engl. J. Med., 213, 918.

GOODALL, J.S. (r920), Practitioner, 105, 37; (1924), Med. Jour. and Rec., $119,14$.

GRAYBIEL, A., and WHITE, P. D. (1935), Amer. Heart J., 10, 345. HALLOCK, P. (1933), Amer. Heart $J ., 9,196$.

HARRIS, A. W., ROSENBAUM, F. F., and ENGLAND, A. C. (1939), Amer. Heart J., 18, 759.

HAY, J. ( 1936$)$, Lancet, ii, I377.

HIMS'WORTH, H. P. (1944), Brit. Med. J., 1, 852; (1944), Brit. Encycl. Med. Pract. Interim Suppl., 25, 14.

JOLL, C. (I932), Diseases of the thyroid gland, London.

KEPLER, E. J., and BARNES, A. R. (I932), Amer. Heart J., 8, ro2.

LERMAN, J. (1932), Amer. Heart J., 8, 146.

LEWIS, J. K., and MCEACHERN,' D. (I93I), Bull. Johns Hopkins Hos., $48,228$.

LIKOFF, W. B., and LEVINE, S. A. (1943), Amer. J. Med. Sci.,

206, 425.

MENARD, O. J., and HURXTHAL, L. M. (1932), Amer. Heart J.,

8, I42.

PARKINSON, J., and COOKSON, H.' (r93I), Quart. J. Med., 24, 499. PEEL, A. A.'F.' (1943), Brit. Heart J., 5, 89 .

RAKE, G., and MCEACHERN, D. (I932), Amer. Heart. J., 8, 19.

RASMUSSEN, H. (194I), Acta Med. Scand. Suppl., I 5 .

READ, J. M.'(1932), Amer. Heart J., 8, 84.

SLESINGER, E. G. (I939), Brit. Med. J., i, 65.

WELLER, C. V., WANTSROM, R. C., GÖRDON, H., and BUGHER,

WILLIUS, F., and HAINES, S. (1925), Amer. Heart J., 1, 67

WILLIUS, F., and HAINES, S. (1925),

ZONDEK, H. (1918), Munch. Mat. Woch., 65, I 180. 